\title{
Effectiveness of Selective Transcatheter Arterial Embolization in Delayed Postpartum Hemorrhage
}

\author{
Se Hwan Kwon ${ }^{1}$, Yeo Jin Kim ${ }^{1}$, Jehong Yoon ${ }^{1}$, Joo Hyeong $\mathrm{Oh}^{1}{ }^{1}$, Myung Gyu Song ${ }^{2}$ and Tae-Seok Seo ${ }^{2, *}$ \\ ${ }^{1}$ Department of Radiology, Kyung Hee University Hospital, College of Medicine, Kyung Hee University, Seoul, Republic of Korea \\ ${ }^{2}$ Department of Radiology, Korea University Guro Hospital, Korea University College of Medicine, Seoul, Republic of Korea \\ "Corresponding author: Department of Radiology, Korea University Guro Hospital, Korea University College of Medicine, Seoul, Republic of Korea. Email: g1q1papa@korea.ac.kr \\ Received 2018 May 10; Revised 2019 January 27; Accepted 2019 January 30.
}

\begin{abstract}
Background: Postpartum hemorrhage (PPH) remains a major cause of maternal morbidity and mortality worldwide. Delayed PPH has received less attention compared to early PPH because the incidence rate of delayed PPH is relatively lower than that of early PPH.

Objectives: The aim of this study was to evaluate the clinical manifestations, angiographic findings, and effectiveness of selective transcatheter arterial embolization (TAE) for delayed PPH.

Patients and Methods: Selective TAE was performed on 37 women (mean age: 32.1 years, range: 24 - 40) with delayed PPH between March 2006 and October 2016. Delayed PPH was defined as continuous hemorrhage from the female genital tract 24 hours to 6 weeks after delivery. Medical records were retrospectively reviewed for patient characteristics, the time interval between delivery and TAE, angiographic findings, embolized artery, embolic materials, technical and clinical success rate, and complications.

Results: Delayed bleeding developed at a median 11.6 days (range: 2 - 34 days) after cesarean section $(n=19)$ and vaginal delivery $(n=$ 18). On angiography, diffuse hypervascularity was detected in 24 patients, pseudoaneurysm in 11, active extravasation in 1, and arteriovenous malformation in 1 . Arteries subjected to TAE included bilateral uterine arteries (UAs) $(n=29)$, bilateral UAs and unilateral ovarian artery $(\mathrm{OA})(\mathrm{n}=4)$, bilateral UAs and bilateral OAs $(\mathrm{n}=1)$, bilateral UAs and cervicovaginal branch $(\mathrm{n}=1)$, unilateral OA $(\mathrm{n}=$ 1), and internal pudendal artery $(n=1)$. Embolic materials were Gelfoam $(n=30)$, Gelfoam and coil $(n=3)$, polyvinyl alcohol (PVA) ( $n$ $=2)$, and PVA and coil $(n=2)$. There were no procedure-related major complications. Technical success was obtained in all patients $(100 \%)$, and clinical success was obtained in 34 patients $(91.9 \%)$

Conclusion: Selective TAE in patients with delayed PPH is a useful treatment with high technical and clinical success.
\end{abstract}

Keywords: Postpartum Hemorrhage, Transcatheter Embolization, Interventional Radiology, Angiography

\section{Background}

In the last few decades, mortality and morbidity rates due to postpartum hemorrhage ( $\mathrm{PPH}$ ) appear to have significantly declined (1-3). However, PPH is still a significant cause of postpartum maternal death and mortality. Generally, PPH is defined as estimated blood loss $>500 \mathrm{~mL}$ or a $\geq 10 \%$ decrease in hematocrit after delivery (4). Severe PPH is responsible for almost $25 \%$ of maternal deaths worldwide $(3,5)$. PPH reportedly occurs in $>4 \%-6 \%$ of all deliveries, and is a significant cause of pregnancy-related maternal complications $(6,7)$. If PPH occurs during the first 24 hours following delivery, it is termed primary or early $\operatorname{PPH}(8,9)$. Secondary, late, or delayed PPH is defined as any hemorrhage from the female genital tract occurring $>24$ hours and within 6 weeks of labor (10). Delayed PPH commonly occurs 8 - 14 days after delivery, with a reported in- cidence rate of $0.5 \%-1.3 \%(5,9,11)$. General causes of delayed PPH include infection, sub-involution of the placental implantation site, and retained products of conception $(11,12)$. Therefore, curettage is a diagnostic and a therapeutic method in the treatment of delayed PPH (9). However, continuous delayed PPH can be lethal and needs surgical or endovascular management (13). Recently, there is increasing interest in the treatment of delayed PPH because of decreasing maternal mortality rates $(9,10,13)$.

Transcatheter embolization (TAE) of the uterine arteries is a well-known procedure for the management of PPH. TAE is a quick and repeatable noninvasive treatment. TAE can be performed with local anesthesia for intractable bleeding $(5,9)$. Therefore, this procedure should be considered as soon as blood transfusions are in progress. Moreover, TAE preserves the uterus, although its effect on fu- 
ture menstruation and pregnancy remains unclear $(14,15)$. However, compared with primary or early PPH, published data for TAE in delayed PPH are limited (8-10).

\section{Objectives}

This study aimed to evaluate the clinical manifestations, angiographic findings, and effectiveness of selective TAE for delayed PPH and to compare and validate the results of previous studies.

\section{Patients and Methods}

Our institutional review board (IRB) approved the protocol of our study, and informed consent was waived because of the retrospective design. Outcomes of 37 women with uncontrollable delayed PPH who were referred for diagnostic angiography and possible management with selective TAE despite obstetric management were retrospectively analyzed. The women were referred to the interventional radiology department between March 2006 and October 2016 in two university hospitals. A total of 200 patients underwent TAE for PPH during this period, of whom 37 presented with delayed PPH (37/200,18.5\%). Delayed PPH was defined as bleeding from the female genital tract between 24 hours and 6 weeks after delivery. The mean patient age was $32.1 \pm 3.9$ years (standard deviation [SD]; age range, 24 - 40 years). Table 1 summarizes obstetric characteristics of our patients.

Digital subtraction pelvic and bilateral internal iliac angiography were performed to find the potential bleeding arteries by one of four interventional radiologists with 4 - 27 years of clinical practice in each institution. A 5-F or 6 -F vascular sheath was placed in the right or left common femoral artery. A 5-F cobra-shaped or reversed J-shaped angiographic catheter (Terumo, Tokyo, Japan) was inserted into the iliac artery or low abdominal aorta over a 0.035inch guidewire under fluoroscopic guidance to select both internal iliac arteries. A microcatheter (Masters Parkway, Asahi Intec, Seto, Japan) was needed for further advancement into the uterine arteries. Diagnostic angiographic findings demonstrated hypertrophied vessels without apparent bleeding foci or active bleeding foci of uterine arteries or other branches of the internal iliac artery. Active contrast medium extravasation into the uterine cavity or pseudoaneurysm formation without extravasation was considered to represent active bleeding sign. Routine bilateral uterine artery TAE was performed even if only hypertrophied uterine arteries were observed, without definite signs of active bleeding, such as contrast extravasation or pseudoaneurysm formation, because of rebleeding risk due to cross-side uterine anastomosis.

Embolization of both uterine arteries was performed using temporary embolic materials. Gelatin sponge particles (Gelfoam) as slurry or pledgets were the first choice of embolic material. Gelfoam was slowly injected into the artery using a microcatheter until near stasis or occlusion was obtained on final angiography. In active bleeding cases (active contrast extravasation or pseudoaneurysm), polyvinyl alcohol (PVA) foam particles (size: 500 - $710 \mu \mathrm{m}$ or 710 - $1000 \mu \mathrm{m}$; Contour, Boston Scientific, Natick, MA, USA) or Micro-coils (Tornado; Cook Medical, Bloomington, IN, USA) were also used as primary or additional embolic agents at the discretion of the interventional radiologist to prevent recanalization of bleeding vessels or to permanently occlude the active extravasations (including pseudoaneurysms).

Completion pelvic or internal iliac artery angiograms were performed in all patients after bilateral uterine artery embolization to evaluate other potential bleeding sites and to confirm no further active bleeding. An abdominal aortogram using a pigtail-shaped catheter was performed in cases with continued hemorrhage after successful TAE of both uterine arteries, to find the ovarian or other arteries, such as the cervicovaginal artery or round ligament arteries from the external iliac artery or other iliac artery branches. Selective embolization of other target arteries was performed when a bleeding focus was suspected on diagnostic angiography.

We assessed patient characteristics, the cause of bleeding, bleeding onset after delivery, details regarding the TAE procedure (embolization materials, arteries selected for embolization, angiographic findings), complications related to TAE, and clinical and technical success. We classified complications into major and minor types as defined in guidelines by the Standards of Practice Committee of the Society of Interventional Radiology (16). According to the guidelines, the classification system is as follows: Minor complications [A - No therapy, no consequence. B Nominal therapy, no consequence]; Major complications [C - requires therapy, minor hospitalization ( $<48$ hours), $\mathrm{D}$ - requires major therapy, unplanned increase in level of care, prolonged hospitalization ( $>48$ hours), E - permanent adverse sequelae, $\mathrm{F}$ - Death].

We defined technical success as successful embolization of all target arteries during the procedure and clinical success as cessation of vaginal hemorrhage or delayed PPH with no further major treatment including additional embolization or surgery before discharge. Results were compared between successful and failed embolization for delayed PPH. Fisher's exact test and the chi-square test were 


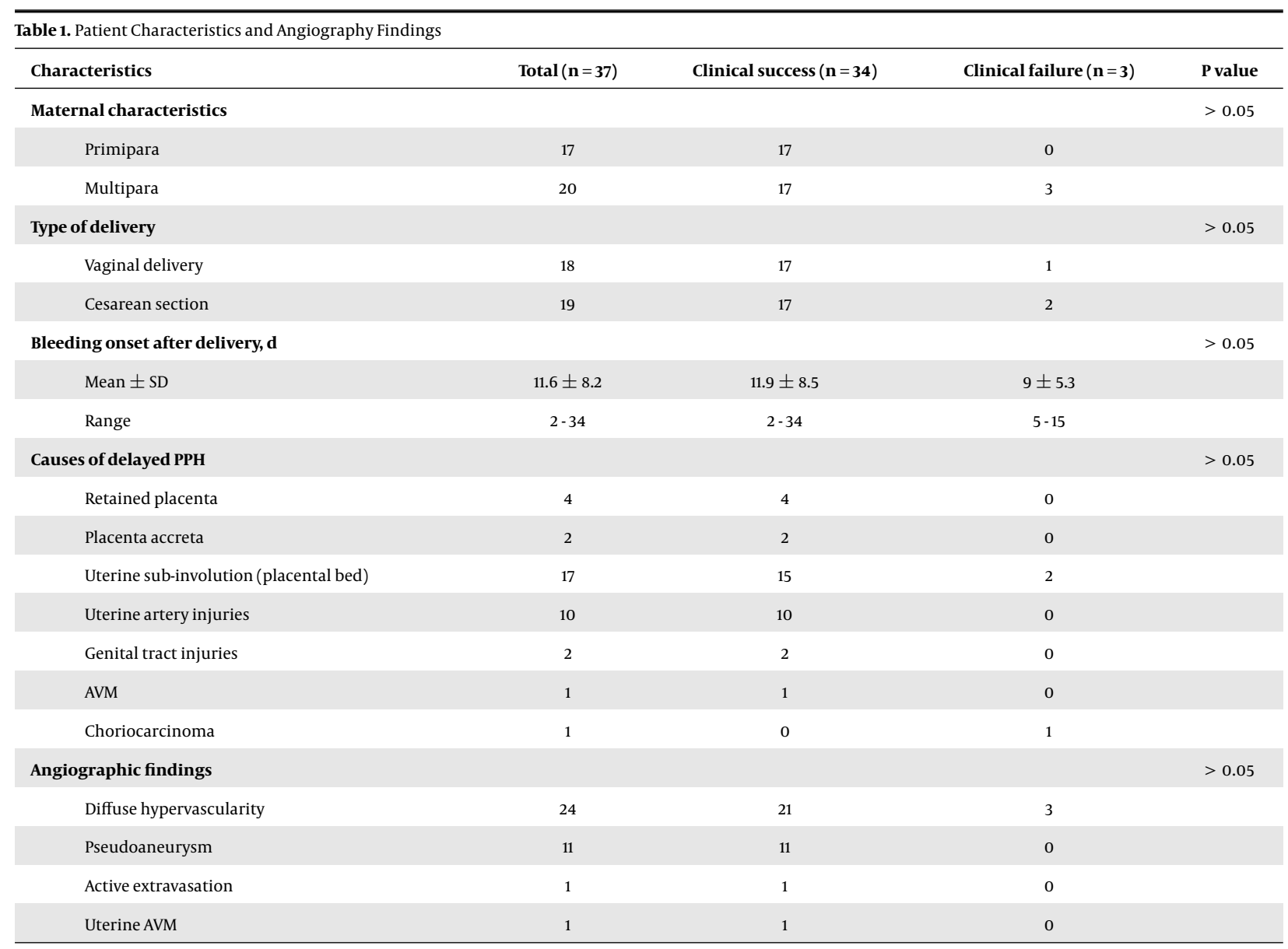

Abbreviations: AVM, arteriovenous malformation; PPH, postpartum hemorrhage; SD, standard deviation; d,days

used (with the DBSTAT program, version 4.1; DBSTAT Co., Chunchon, Korea). Statistical significance was set at $\mathrm{P}<$ 0.05 .

\section{Results}

TAE was performed 2 - 34 days after labor (mean 11.6 \pm 8.2 days) in 37 women with uncontrollable delayed $\mathrm{PPH}$ who were referred for diagnostic pelvic angiography and possible management with selective TAE. Of these patients, 17 (17/37, 45.9\%) were primiparous, and 20 (20/37, 54.1\%) were multiparous. Among 17 primiparas, 10 had vaginal deliveries (10/17, 58.8\%) and seven had cesarean sections (7/17, 41.2\%). Among 20 multiparas, eight had vaginal deliveries (8/20, 40\%) and 12 had cesarean sections (12/20, 60\%). Sixteen multiparas had two pregnancies with delivery (16/30, $80 \%$ ), and four multiparas had three pregnancies with delivery (4/20,20\%). Retained placental tissue was diagnosed in four patients $[4 / 37,10.8 \%$; vaginal deliveries $(n=2)$, cesarean section $(n=2)$, and placenta accreta was diagnosed in two [2/37, 5.4\%; vaginal deliveries $(\mathrm{n}=2)]$. No other patients had a placental abnormality (31/37; 83.8\%). Placental abnormalities were diagnosed with ultrasonography. All patients were referred to interventional radiology because they did not respond to obstetric management including administration of intravenous fluid, transfusion, uterotonic agents, or dilatation and curettage for remnant placental tissue.

At the time of initial admission and diagnosis, 27 patients were medically managed before TAE was considered because they were hemodynamically stable. However, 10 patients were hemodynamically unstable before TAE, with tachycardia (heart rate $>100$ ) or hypotension ( $<90 \mathrm{mmHg}$ systolic blood pressure). Blood transfusion was performed for significant blood loss and unstable hemodynamic status.

The most frequent angiographic findings of delayed PPH were diffuse hypervascularity without active bleeding signs ( $n=24 ; 64.9 \%$ ), followed by pseudoaneurysm $(n=11$; 
29.7\%), uterine arteriovenous malformation (AVM) ( $\mathrm{n}=1$; $2.7 \%$ ) and active extravasation $(n=1 ; 2.7 \%)$ (Figures 1 and 2 ). As noted, pseudoaneurysm formation and active extravasation were regarded as active bleeding signs and other findings were regarded as indirect bleeding signs. Delayed PPH developed at a median 11.6 days (range: 2 - 34 days) after cesarean section $(n=19)$ and vaginal delivery $(n=18)$. TAE was used for the following: bilateral uterine arteries ( $\mathrm{n}=$ 29), bilateral uterine arteries and unilateral ovarian artery $(\mathrm{n}=4)$, bilateral uterine arteries and bilateral ovarian arteries $(n=1)$, bilateral uterine arteries and cervicovaginal branch $(n=1)$, unilateral ovarian artery $(n=1)$, and internal pudendal artery $(n=1)$ (Table 2$)$. Embolic materials were mostly Gelfoam $(n=30)$, Gelfoam and coil $(n=3)$, PVA ( $n$ $=2)$, and PVA and coil $(n=2)$. There were no procedurerelated major complications.

\begin{tabular}{ll}
\hline Table 2. Arteries Embolized During Procedures & \\
\hline Total & No. of patients $(\mathbf{n}=\mathbf{3 7})$ \\
\hline Patients with active bleeding signs $(\mathbf{n}=\mathbf{1 2})$ & 9 \\
\hline Bilateral UAs & 1 \\
\hline Bilateral UAs + left cervicovaginal branch & 1 \\
\hline Right internal pudendal artery & 1 \\
\hline Left UA & \\
\hline Patients without active bleeding signs $(\mathbf{n}=\mathbf{2 5})$ & 20 \\
\hline Bilateral UAs & 2 \\
\hline Bilateral UAs + left OA & 2 \\
\hline Bilateral UAs + right OA & 1 \\
\hline Bilateral UAs + bilateral OAs
\end{tabular}

Abbreviations: OA, ovarian artery; UA, uterine artery

Embolization was technically successful in all 37 patients (100\%) (Figure 3). The first session of embolization was clinically successful in 34 (91.9\%) patients. In two of three clinical failure cases, repeated TAE was successful. Therefore, the total clinical success rate of all sessions of TAE was $97.3 \%$ (36 of 37 patients). However, one of three clinical failure cases presented continued vaginal bleeding and pelvic pain even after secondary TAE. Choriocarcinoma was diagnosed on pelvic magnetic resonance image (MRI) (Figure 4) and laparoscopically assisted vaginal hysterectomy (LAVH) was performed in this patient. Six cycles of chemotherapy with methotrexate were performed. At the time of writing this article, the patient has been monitored for 6 years and continues to be in good physical condition without cancer recurrence or significant symptoms.

Four patients were not followed up after hospital discharge, and were reported as "lost to follow-up." For the 32 overall clinical success patients with regular follow-up, the median and mean follow-up durations were 2 months and 7.6 months (range, 1- 95 months), respectively. Regular menstrual cycles resumed in all patients during the followup period. Three women became pregnant and gave birth without complications. Medical records did not indicate how many patients intended to become pregnant after TAE for delayed PPH. However, no patients complained of infertility during the follow-up period in our records. Morbidity and mortality were not reported in all patients. No statistical association was observed between clinical success of TAE for delayed PPH and any of the analyzed factors (P > $0.05)$.

\section{Discussion}

PPH remains a major cause of maternal morbidity and mortality worldwide (7). However, delayed PPH has received somewhat less attention compared to early PPH because the incidence rate of delayed PPH is relatively lower than that of early PPH, and delayed PPH is usually associated with maternal morbidity rather than mortality. Moreover, clinicians often miss potential bleeding because delayed PPH usually occurs during the 8 - 14 days after delivery. Patients are usually discharged from the hospital by that time $(6,9,13)$. However, continuous and severe delayed PPH is dangerous and life-threatening because of hypovolemic shock. Usually, general causes of delayed PPH are infection, retained products of conception, and sub-involution of the placental implantation site $(1,11)$. Other causes are vascular abnormalities affecting the myometrial circulation (arterial injuries, such as pseudoaneurysm and AVM).

Some authors have examined the clinical effectiveness and safety of embolization for delayed PPH and have documented clinical TAE success rates of $>90 \%$ for delayed PPH $(6,7)$. Park et al. evaluated 52 women with delayed $\mathrm{PPH}$, and showed a high clinical success rate (90.9\%) and no procedure-related significant complications (10). Our retrospective analysis showed that selective TAE in patients with delayed PPH is a useful treatment with high technical and clinical success. The technical success rate was $100 \%$ (37/37) and the total clinical success rate for all sessions of TAE, both primary and additional sessions, was 97.3\% (36 of 37 patients). Generally identified causes of repeat bleedings after TAE include arterial spasm, collateral vessels, and placental abnormality (17). However, compared with early PPH, published data on TAE for delayed PPH are still limited (8-10). Previous studies of TAE for early PPH with 40 or more patients reported clinical success rates ranging from $71.5 \%$ - 89.1\%. Lee et al. (5) reported results for a larger sample size in which the overall clinical success rate, with both initial 

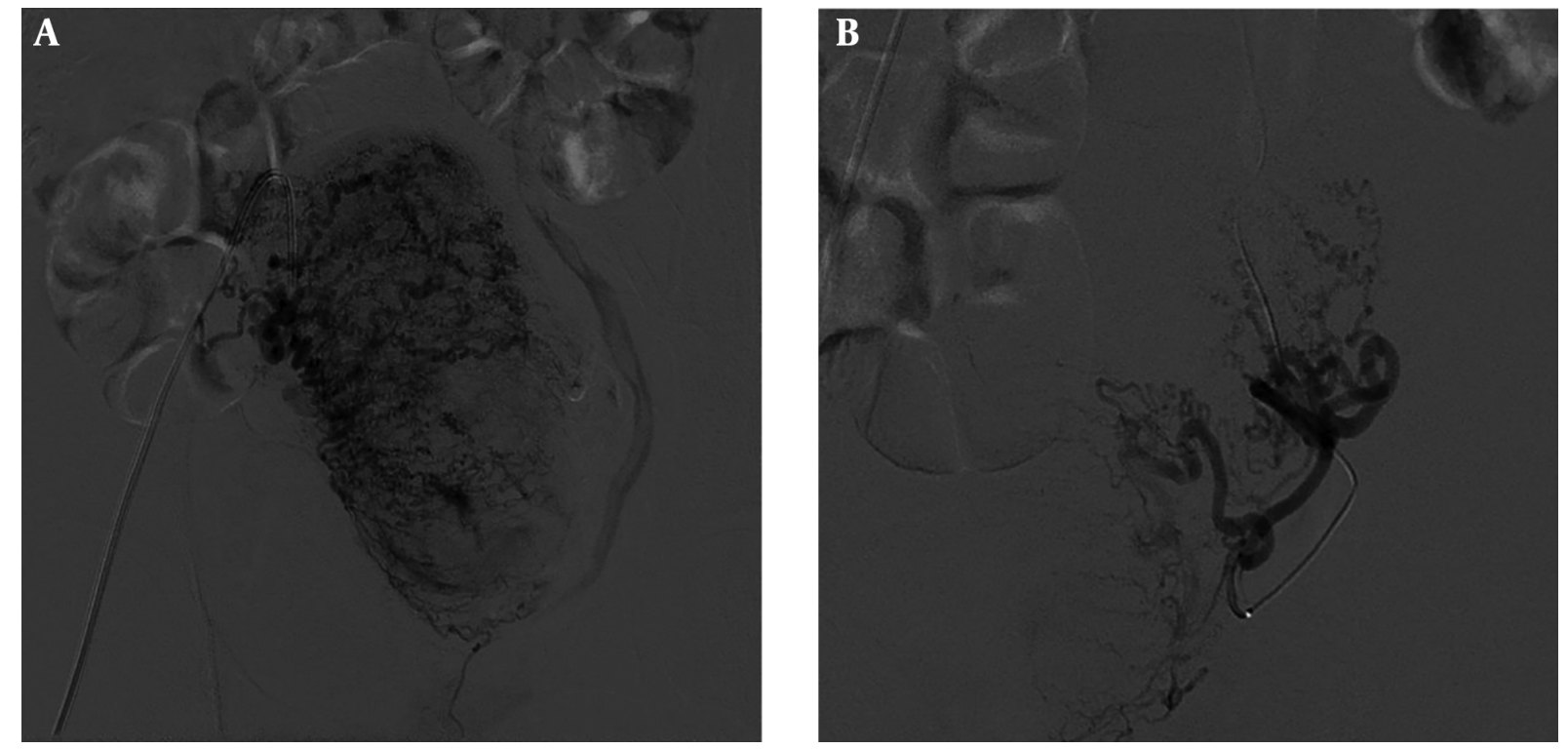

Figure 1. A 40-year-old woman with vaginal bleeding 8 days after vaginal delivery. Right (A) and left (B) internal iliac angiography shows tortuous uterine arteries and prominent uterine vascularity without definite active bleeding. Embolization of both uterine arteries was performed using gelatin sponge particles and polyvinyl alcohol (PVA).

and repeat TAE, was 90.4\% (227 of 251) and overall bleeding control was achieved in 98\% (246/251) in early or primary $\mathrm{PPH}$ patients. More studies are needed on management of delayed PPH because the incidence rate of delayed PPH is relatively low as compared with early PPH.

In our study, only 1 clinical failure case presented with continued vaginal bleeding and pelvic pain, even after secondary TAE. Choriocarcinoma was diagnosed on pelvic MRI and LAVH was performed in this patient. Full-term pregnancy with choriocarcinoma is a rare disease (18) that may present as intrauterine fetal death, hydrops fetalis, fetomaternal hemorrhage, or PPH. However, diagnosis of gestational choriocarcinoma after a full-term pregnancy is not easy without a histopathological study of the uterus or placenta. The low incidence rate of gestational choriocarcinoma after term pregnancy does not result in a routine histopathological study of the uterus and placenta after birth in all hospitals $(2,19)$. The prognosis of women diagnosed with choriocarcinoma is not bad if an appropriate chemotherapeutic drug is started early. However, delayed diagnosis and treatment affect the result by increasing the risk of distal metastasis and increased resistance to chemotherapy treatment. It is essential to be aware of delayed PPH, and postpartum choriocarcinoma should be included in the differential diagnosis of uncontrolled delayed PPH. In our patient, six cycles of chemotherapy with methotrexate were performed. With monitoring for 6 years, there has been no cancer recurrence or significant symptoms.

Ultrasound is a quick and noninvasive diagnostic tool for PPH. Some authors have advocated ultrasound study of the uterine cavity to find retained placental tissue while others have found that ultrasound for retained placental tissue was not particularly accurate $(13,20,21)$. A pseudoaneurysm of uterine or genital tract arteries has been reported as an outcome of procedures such as dilatation and curettage, hysterectomy, cesarean section, myomectomy, oocyte retrieval for in-vitro fertilization, and even routine vaginal delivery (22). Uterine arterial active extravasation and rupture of a pseudoaneurysm are life-threatening events in delayed PPH, requiring early diagnosis and treatment. In an emergency setting, color-Doppler ultrasound may demonstrate a pseudoaneurysm. Computed tomography or MRI can also make the diagnosis and rule out other causes of delayed PPH. However, conventional angiography remains the standard for definitive diagnosis and can provide adequate treatment $(1,5,9,10)$.

In the past, appropriate management of delayed PPH remained unclear. Traditional methods included administration of ergometrine with or without oxytocin. Prostaglandins have been advocated (13), but there is not yet enough evidence to indicate clear effectiveness. Although curettage is useful in the majority of women with retained placental tissue, some patients continue to have bleeding. More curettage may be helpful, but the chances of uterine damage increase (13). While uterine cavity tam- 

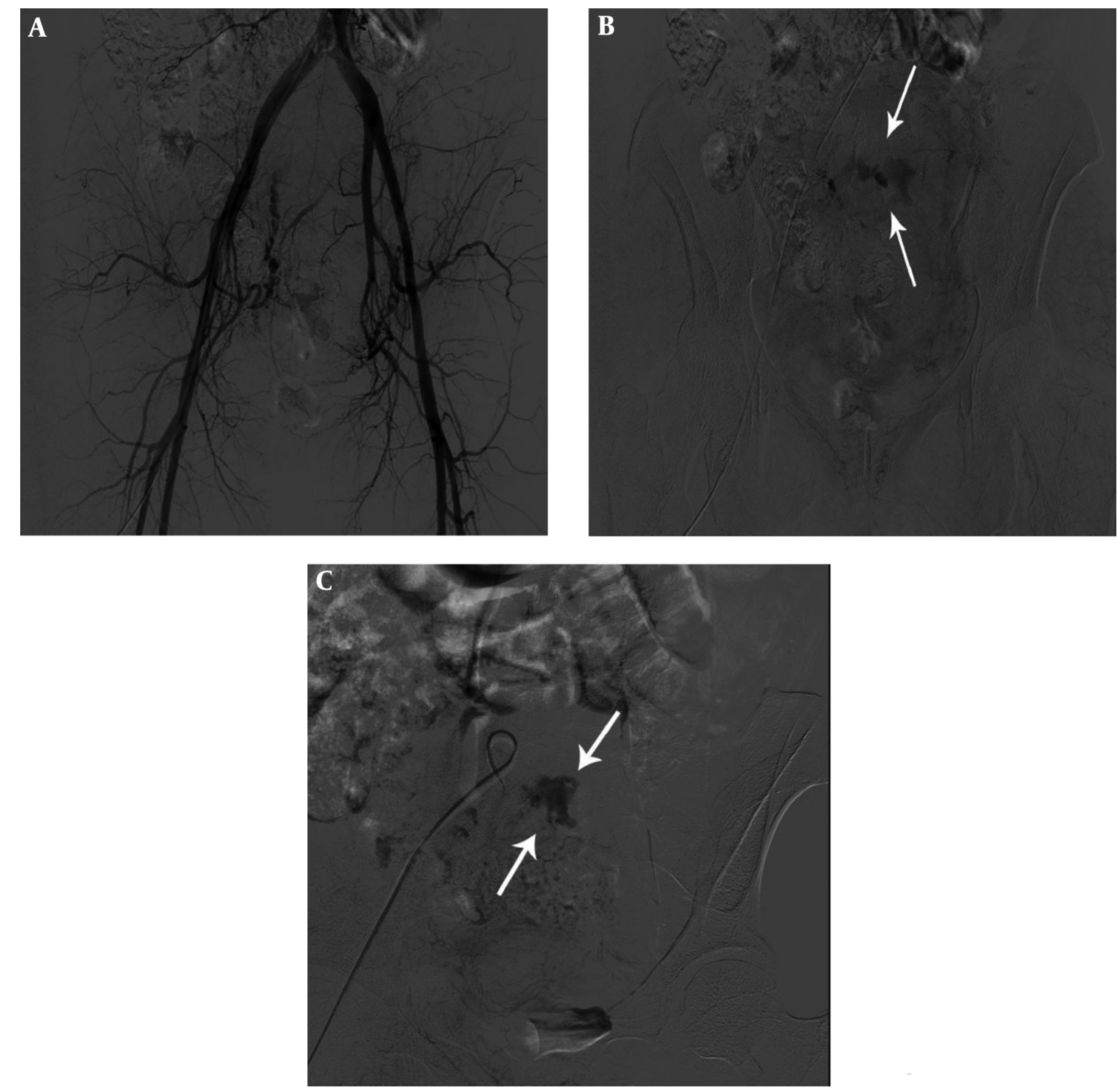

Figure 2. A 39-year-old woman with massive PPH 10 days after cesarean section. Early (A) and delayed pelvic angiography (B) and right internal iliac angiography (C) show prominent right uterine arteries and active extravasation of contrast medium (arrows). Embolization of the right uterine artery was subsequently performed using gelatin sponge particles and micro-coils.

ponade with uterine balloons (23) or other aggressive surgical treatment including total hysterectomy or internal iliac vessel ligation may be necessary, a more recent option, TAE of uterine or other arteries, might now be considered $(9,10)$. TAE has clear advantages in that it is less invasive and does not need general anesthesia in a probably unstable patient. Moreover, it is easy to determine the origin of hemorrhage, leading to prompt control of bleeding, a low incidence rate of rebleeding, and a high success rate (> 90\% for delayed PPH). Although TAE has many advantages, some complications can arise, such as postembolization syndrome, groin hematoma or pseudoaneurysm formation, ischemic tissue changes or abscess formation, and contrast-induced nephrotoxicity (24). Fortunately, there were no procedure-related or other major complications in our study. 


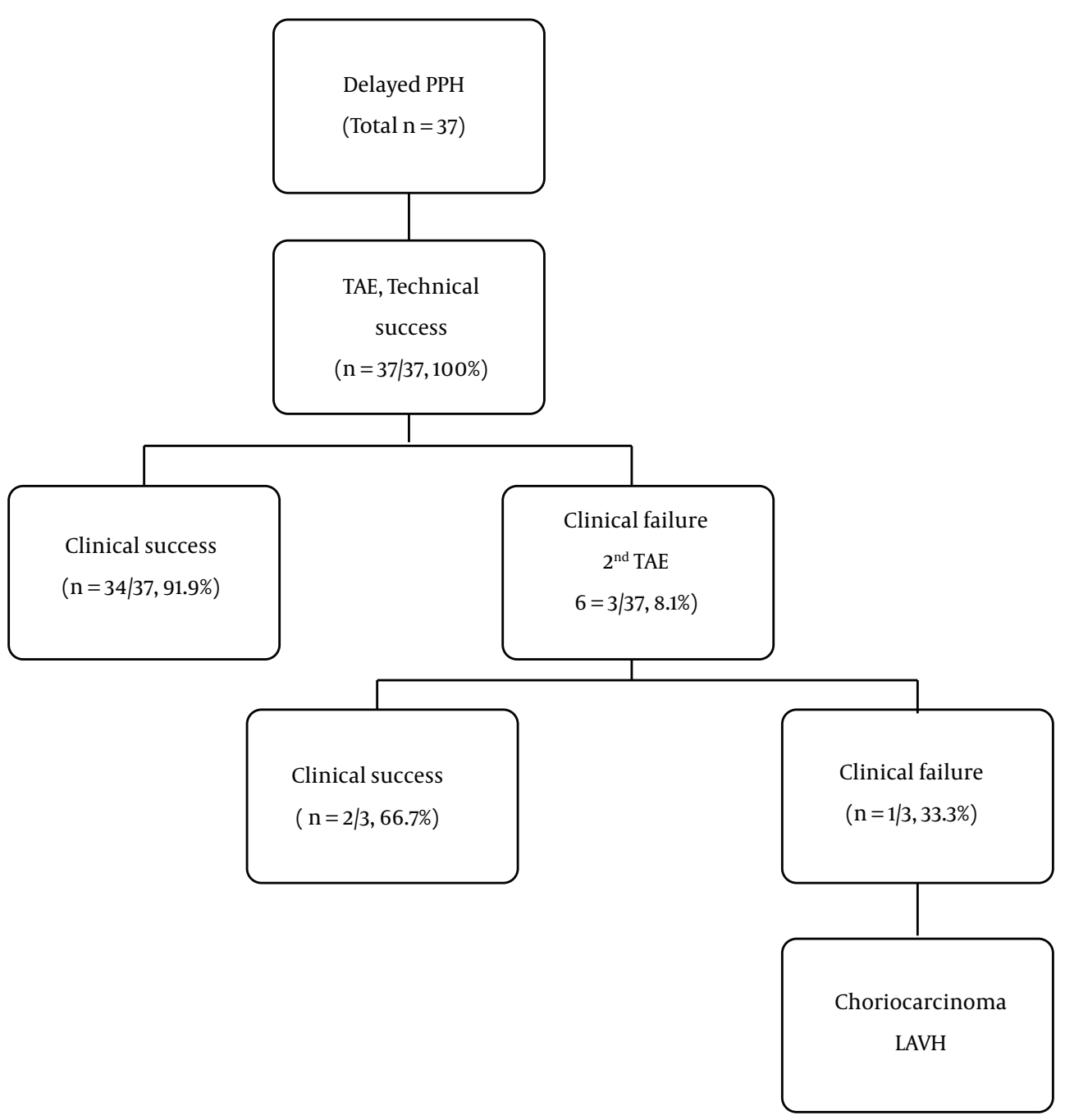

Figure 3. Flow chart shows results of TAE for delayed PPH. (PPH, postpartum hemorrhage; TAE, transcatheter arterial embolization; LAVH, laparoscopically-assisted vaginal hysterectomy).

Park et al. (10) reported that clinical success of TAE for delayed PPH was not related to any of the analyzed factors. Similarly, in our study, there was no statistical association between the clinical success of TAE for delayed PPH and any of the analyzed factors ( $P>0.05)$, such as maternal characteristics, type of delivery, bleeding onset after delivery, causes of delayed PPH, and angiographic findings. However, we think that more studies are needed to identify the risk factors for clinical failure after TAE because the sample size of our study and Park's study is small and a nonsignificant p-value may be due to low sample size in subgroups.

All women with available follow-up (clinical success cases) in our study resumed regular menstrual cycles, and 3 became pregnant and gave birth without complications during the follow-up period. No patients complained of infertility during the follow-up period in our records. However, the impact of TAE on menstrual cycles or fertility has not been studied sufficiently, and there are no explicit methods that can determine whether future fertility or pregnancies would be affected by TAE. Some researchers reported a higher incidence and risk of placental abnormalities and fetal growth restriction (25). However, Hardeman et al. (26) reported no statistically significant differences in pregnancy or fertility between women who underwent 

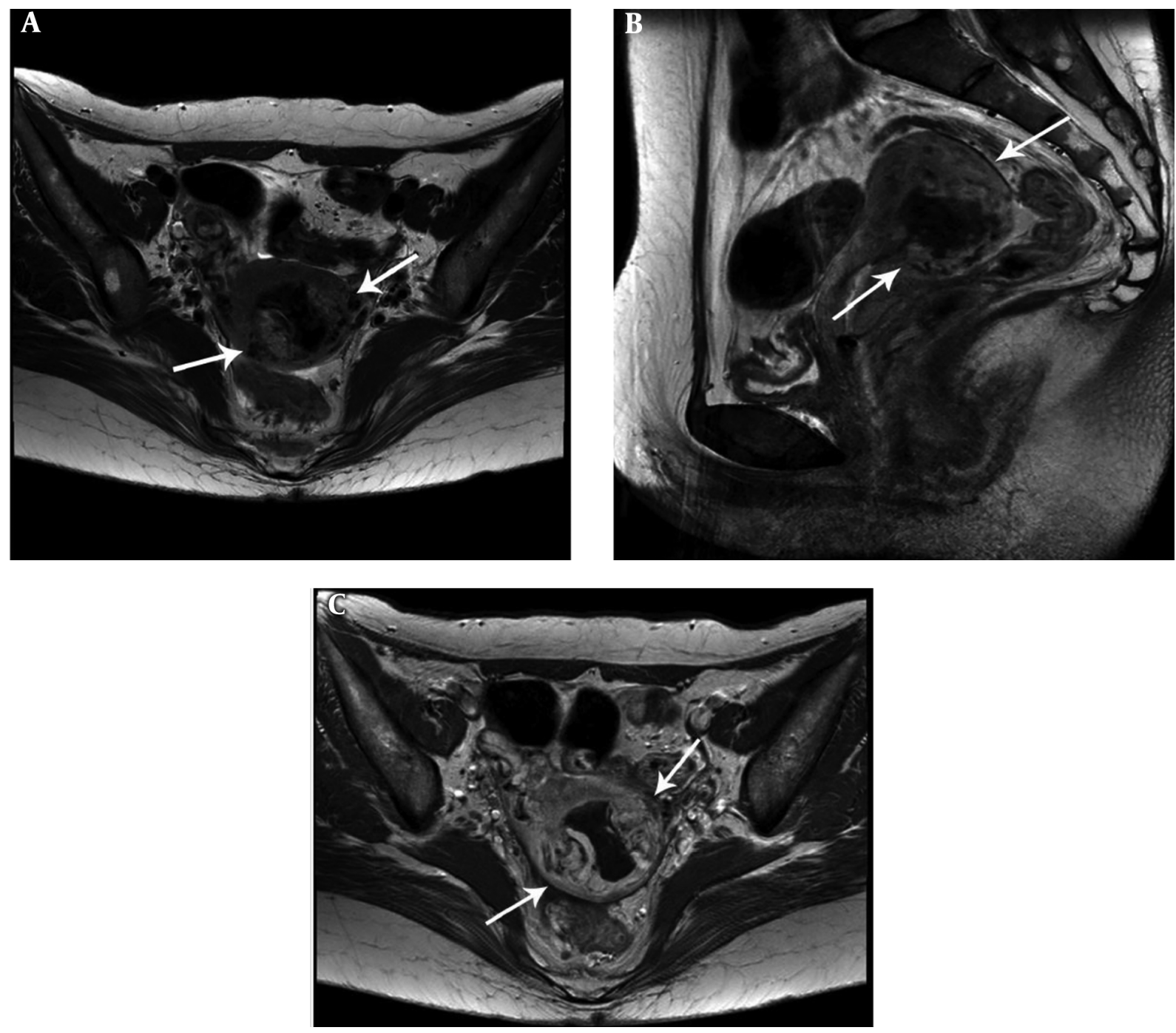

Figure 4. A 30-year-old woman with vaginal bleeding 15 days after vaginal delivery. Continued vaginal bleeding was noted even after secondary transcatheter arterial embolization [TAE]. Choriocarcinoma was diagnosed with pelvic magnetic resonance imaging. T2-weighted axial (A), sagittal (B) and contrast-enhanced T1-weighted axial images (C) show a 5.1-cm irregular shaped mass in the posterior body of the uterus (arrows) with central necrosis.

uterine artery embolization for PPH and those who did not.

Our study had some limitations, one of which was the possibility of recall bias caused by the retrospective examination of medical charts or records. Moreover, TAE procedures are controversial and necessarily subjective because there are no evidence-based standard guidelines for TAE in delayed PPH. Another limitation of this study was irregular follow-up by patients, as some women did not visit the hospital as recommended. Further studies on the most effective treatment procedures and standard guidelines for TAE techniques are needed. In addition, long-term followup study of ovarian function and fertility is needed.

In conclusion, selective TAE for delayed PPH is an ef- fective and safe treatment with high technical and clinical success rates. Above all, TAE can preserve fertility. We recommend selective TAE as the initial treatment of choice for delayed PPH.

\section{Footnotes}

Authors' Contributions: Study concept and design: Se Hwan Kwon, Yeo Jin Kim and Tae-Seok Seo; acquisition of data: Se Hwan Kwon, Yeo Jin Kim, Jehong Yoon and TaeSeok Seo; analysis and interpretation of data: Se Hwan Kwon, Yeo Jin Kim, Jehong Yoon, Joo Hyeong Oh, Myung Gyu Song and Tae-Seok Seo; drafting of the manuscript: 
Se Hwan Kwon, Yeo Jin Kim, Jehong Yoon, Joo Hyeong Oh, Myung Gyu Song and Tae-Seok Seo; critical revision of the manuscript for important intellectual content: Se Hwan Kwon, Yeo Jin Kim and Tae-Seok Seo; statistical analysis: Jehong Yoon and Joo Hyeong Oh; study supervision: Tae-Seok Seo

Conflict of Interests: None declared.

Ethical Approval: 2017-06-022-004, KHMC IRB.

Funding/Support: The authors declare no funding/support.

\section{References}

1. Yun SY, Lee DH, Cho KH, Lee HM, Choi YH. Delayed postpartum hemorrhage resulting from uterine artery pseudoaneurysm rupture. J Emerg Med. 2012;42(1):e11-4. doi: 10.1016/j.jemermed.2011.03.005. [PubMed: 21497477].

2. Ryu N, Ogawa M, Matsui H, Usui H, Shozu M. The clinical characteristics and early detection of postpartum choriocarcinoma. Int J Gynecol Cancer. 2015;25(5):926-30. doi: 10.1097/IGC.0000000000000184. [PubMed: 24987912].

3. Al-Zirqi I, Vangen S, Forsen L, Stray-Pedersen B. Prevalence and risk factors of severe obstetric haemorrhage. BJOG. 2008;115(10):1265-72. doi: 10.1111/j.1471-0528.2008.01859.x. [PubMed:18715412].

4. Knight M, Callaghan WM, Berg C, Alexander S, Bouvier-Colle MH, Ford $\mathrm{JB}$, et al. Trends in postpartum hemorrhage in high resource countries: A review and recommendations from the International Postpartum Hemorrhage Collaborative Group. BMC Pregnancy Childbirth. 2009;9:55. doi: 10.1186/1471-2393-9-55. [PubMed: 19943928]. [PubMed Central: PMC2790440].

5. Lee HY, Shin JH, Kim J, Yoon HK, Ko GY, Won HS, et al. Primary postpartum hemorrhage: Outcome of pelvic arterial embolization in 251 patients at a single institution. Radiology. 2012;264(3):903-9. doi: 10.1148/radiol.12111383. [PubMed: 22829685].

6. Carroli G, Cuesta C, Abalos E, Gulmezoglu AM. Epidemiology of postpartum haemorrhage: A systematic review. Best Pract Res Clin Obstet Gynaecol. 2008;22(6):999-1012. doi: 10.1016/j.bpobgyn.2008.08.004. [PubMed: 18819848].

7. Gilbert L, Porter W, Brown VA. Postpartum haemorrhage-A continuing problem. Br J Obstet Gynaecol. 1987;94(1):67-71. doi: 10.1111/j.14710528.1987.tb02255.x. [PubMed: 3493028].

8. King PA, Duthie SJ, Dong ZG, Ma HK. Secondary postpartum haemorrhage. Aust N Z J Obstet Gynaecol. 1989;29(4):394-8. doi: 10.1111/j.1479828X.1989.tb01776.x. [PubMed: 2631675].

9. Pelage JP, Soyer P, Repiquet D, Herbreteau D, Le Dref O, Houdart E, et al. Secondary postpartum hemorrhage: Treatment with selective arterial embolization. Radiology. 1999;212(2):385-9. doi: 10.1148/radiology.212.2.r99jl05385. [PubMed: 10429694].

10. Park HS, Shin JH, Yoon HK, Kim JH, Gwon DI, Ko GY, et al. Transcatheter arterial embolization for secondary postpartum hemorrhage: Outcome in 52 patients at a single tertiary referral center. J Vasc Interv Radiol. 2014;25(11):1751-7. doi: 10.1016/j.jvir.2014.05.009. [PubMed: 24985718].

11. Khong TY, Khong TK. Delayed postpartum hemorrhage: A morphologic study of causes and their relation to other pregnancy disorders. Obstet Gynecol.1993;82(1):17-22. [PubMed: 8515920].
12. Cooper BC, Hocking-Brown M, Sorosky JI, Hansen WF. Pseudoaneurysm of the uterine artery requiring bilateral uterine artery embolization. J Perinatol. 2004;24(9):560-2. doi: 10.1038/sj.jp.7211119. [PubMed: 15329736].

13. Hoveyda F, MacKenzie IZ. Secondary postpartum haemorrhage: Incidence, morbidity and current management. BJOG. 2001;108(9):92730. [PubMed: 11563461].

14. Salomon LJ, deTayrac R, Castaigne-Meary V, Audibert F, Musset D, Ciorascu R, et al. Fertility and pregnancy outcome following pelvic arterial embolization for severe post-partum haemorrhage. A cohort study. Hum Reprod. 2003;18(4):849-52. doi: 10.1093/humrep/deg168. [PubMed: 12660283].

15. Descargues G, Mauger Tinlot F, Douvrin F, Clavier E, Lemoine JP, Marpeau L. Menses, fertility and pregnancy after arterial embolization for the control of postpartum haemorrhage. Hum Reprod. 2004;19(2):339-43. doi: 10.1093/humrep/deh082. [PubMed: 14747177].

16. Sacks D, McClenny TE, Cardella JF, Lewis CA. Society of interventional radiology clinical practice guidelines. J Vasc Interv Radiol. 2003;14(9 Pt 2):S199-202. doi: 10.1097/01.RVI.0000094584.83406.3e. [PubMed: 14514818].

17. Soyer P, Dohan A, Dautry R, Guerrache Y, Ricbourg A, Gayat E, et al. Transcatheter arterial embolization for postpartum hemorrhage: Indications, technique, results, and complications. Cardiovasc Intervent Radiol. 2015;38(5):1068-81. doi: 10.1007/s00270-015-1054-y. [PubMed: 25677130].

18. Peng HH, Ng ZP, Tang YH, Chua AA, Huang KG. Term pregnancy with choriocarcinoma presenting as severe fetal anemia and postpartum hemorrhage. Taiwan J Obstet Gynecol. 2016;55(3):430-3. doi: 10.1016/j.tjog.2016.04.021. [PubMed: 27343330].

19. Bratila E, Ionescu CA, Vladescu CT, Cirstoiu MM, Berceanu C. Gestational choriocarcinoma after term pregnancy: A case report. Rom J Morphol Embryol. 2015;56(1):267-71. [PubMed: 25826515].

20. Skinner J, Turner MJ. Postpartum exploration of the genital tract under general anaesthesia reviewed.JObstet Gynaecol.1997;17(3):273. doi: 10.1080/01443619750113258. [PubMed: 15511848].

21. Lee CY, Madrazo B, Drukker BH. Ultrasonic evaluation of the postpartum uterus in the management of postpartum bleeding. Obstet $G y$ necol.1981;58(2):227-32. [PubMed: 6973109].

22. Kovo M, Behar DJ, Friedman V, Malinger G. Pelvic arterial pseudoaneurysm-a rare complication of Cesarean section: Diagnosis and novel treatment. Ultrasound Obstet Gynecol. 2007;30(5):783-5. doi: 10.1002/uog.5139. [PubMed: 17899575].

23. Bowen LW, Beeson JH. Use of a large Foley catheter balloon to control postpartum hemorrhage resulting from a low placental implantation. A report of two cases. J Reprod Med.1985;30(8):623-5. [PubMed: 3900389].

24. Vedantham S, Goodwin SC, McLucas B, Mohr G. Uterine artery embolization: An underused method of controlling pelvic hemorrhage. Am J Obstet Gynecol. 1997;176(4):938-48. doi: 10.1016/S00029378(97)70624-0. [PubMed: 9125624].

25. McGonegle SJ, Dziedzic TS, Thomas J, Hertzberg BS. Pseudoaneurysm of the uterine artery after an uncomplicated spontaneous vaginal delivery. J Ultrasound Med. 2006;25(12):1593-7. doi: 10.7863/jum.2006.25.12.1593. [PubMed: 17121956].

26. Hardeman S, Decroisette E, Marin B, Vincelot A, Aubard Y, Pouquet M, et al. Fertility after embolization of the uterine arteries to treat obstetrical hemorrhage: A review of 53 cases. Fertil Steril. 2010;94(7):2574-9. doi: 10.1016/j.fertnstert.2010.02.052. [PubMed: 20381035]. 